\title{
Vivienda habitual y empleo: efectos de la crisis económica en España
}

\author{
Javier A. Barrios García \\ Universidad de La Laguna
}

\section{Resumen}

Analizamos el efecto de la forma de tenencia de la vivienda habitual (propiedad/alquiler) sobre la situación de empleo de los individuos (empleado/desempleado) basándonos en datos de panel para España a lo largo del periodo 2004-2013. Teniendo en cuenta la heterogeneidad inobservada, la endogeneidad de la elección de tenencia de vivienda, y distinguiendo la dependencia del estado espuria de la verdadera, encontramos que la tasa de vivienda en propiedad regional no genera ningún efecto indirecto sobre la probabilidad de empleo, mientras que ser propietario de la vivienda habitual aumenta de forma significativa y robusta la probabilidad de empleo en torno a 2 puntos porcentuales, especialmente para los propietarios con hipoteca, desapareciendo este efecto para aquellos sin hipoteca.

Palabras clave: vivienda en propiedad, desempleo.

Clasificación JEL: C33, C35, J64, R23.

\begin{abstract}
We analyze the effect of housing tenure choice (owning/renting) on the employm-ent status of individuals (employed/unemployed) using panel data for Spain over the period 2004-2013. Taking into account unobserved heterogeneity, the endog-eneity of housing tenure choice, and distinguishing between spurious state dependence and a true one, we find that the aggregated regional homeownership rate does not have any significant spillover effect on the probability of employment, while owning a home significantly and robustly increases the likelihood of employment around 2 percentage points, especially for those owners with a mortgage outstanding, vanishing this effect for the outright owners.
\end{abstract}

Keywords: homeownership, unemployment.

JEL classification: C33, C35, J64, R23.

\section{Introducción}

En el discurso económico, tanto dentro del ámbito académico como político, hay un mantra recurrente que recomienda incentivar el sector de vivienda en alquiler con el objetivo de facilitar la movilidad de los trabajadores e incrementar así las posibilidades de empleo. Sin embargo, como veremos a continuación, esta afirmación no viene amparada en el caso español por una contrastación empírica rigurosa, ni desde el punto de vista agregado, ni tampoco a escala microeconómica.

A nivel internacional, tras los trabajos pioneros de Oswald (1996), (1999), una extensa literatura ha dedicado sus esfuerzos al análisis de los efectos que genera el mercado de la vivienda sobre el mercado laboral. En el plano teórico, el modelo 
de búsqueda de empleo en la tradición de Mortensen (1986) o Pissarides (2000) ha sido adoptado por diversos autores para incluir la forma de tenencia de la vivienda habitual como factor adicional que repercute en la situación de empleo del individuo (Dohmen, 2005; Munch et al., 2006, 2008; Coulson y Fisher, 2009; Rouwendal y Nijkamp, 2010; Head y Lloyd-Ellis, 2012). El principal mecanismo de transmisión que postulan la mayoría de estos modelos se fundamenta en los costes de mudanza, argumentándose que los propietarios de vivienda afrontan en general unos costes de mudanza mayores que los inquilinos y, por ende, son menos propensos a mudarse para atender ofertas de trabajo en áreas laborales distintas a la de residencia, incrementando su salario de reserva en caso de mudanza para compensar estos costes adicionales. Sin embargo, bajo este paraguas teórico cabe apuntar también mecanismos de transmisión adicionales, algunos con efectos contrapuestos: las disparidades en el coste de la vivienda que pueden incidir sobre el pago final (utilidad) que recibe el individuo (Coulson y Fisher, 2009; Head y Lloyd-Ellis, 2012), diferencias en la tasa de ofertas de trabajo o en la probabilidad de despido entre propietarios e inquilinos derivadas del llamado «efecto vecindario» (Ioannides y Loury, 2004; Dujardin y Goffette-Nagot, 2010), divergencias en la tasa de descuento temporal entre ambos colectivos (Della Vigna y Paserman, 2005; James, 2009), o incluso en la disposición a mudarse debido a las repercusiones dispares que genera en ambos colectivos la caída de los precios de la vivienda en periodos de crisis económica (Farber, 2012; Coulson y Grieco, 2013).

En el campo empírico, existe una doble evidencia a nivel internacional, tanto a escala macro como micro. Por un lado, varios estudios corroboran la hipótesis de Oswald $(1996,1999)$ de que la tenencia de vivienda en propiedad aumenta la probabilidad de desempleo del individuo, lo cual les lleva a afirmar desde un punto de vista agregado que una mayor tasa de vivienda en propiedad conlleva un incremento de la tasa de desempleo (Nickell, 1998; Nickell y Layard, 1999, siendo esta tesis sustentada también parcialmente en Green y Hendershott, 2001; Brunet y Lesueur, 2009; Isebaert et al., 2015). Por el contrario, una gran mayoría de trabajos a nivel micro ha concluido en el sentido opuesto a la hipótesis de Oswald, asociándose en este caso la vivienda en propiedad a una mayor probabilidad de empleo (Coulson y Fisher, 2002, 2009; Flatau et al., 2003; Munch et al., 2006; Battu et al., 2008; Rouwendal y Nijkamp, 2010). Esta dicotomía se ha reproducido a nivel español, dependiendo del periodo de tiempo analizado. Por una parte, a nivel agregado, Barrios y Rodríguez (2004) niegan la hipótesis de Oswald con datos de las provincias españolas para 1991, corroborado posteriormente en Rodríguez y Barrios (2012) a nivel micro para el año 2010. Los mismos autores (Rodríguez y Barrios, 2013) en un análisis transversal para el periodo 2006-2009 concluyen que no se evidencia influencia alguna de la forma de tenencia de la vivienda sobre la situación de empleo de los individuos. La controversia, por tanto, está servida.

Recientemente, algunos autores han tratado de reconciliar esta disparidad de resultados distinguiendo entre un efecto a nivel micro de la tenencia de vivienda en propiedad sobre la situación de empleo del individuo, de la influencia simultánea que 
pudiera ejercer la tasa de vivienda en propiedad agregada. Así, Blanchflower y Oswald (2013) argumentan que el mercado de vivienda podría generar externalidades sobre el mercado laboral y la economía en general, explicando esto la fuerte correlación existente en los diferentes estados de Estados Unidos entre altos niveles de vivienda en propiedad y las tasas de desempleo en los años subsiguientes. También apuntan algunos mecanismos de transmisión para este efecto: las externalidades informacionales que una mayor movilidad laboral puede generar sobre la productividad de los trabajadores, las restricciones derivadas de la legislación en planeamiento y edificación vigente que podrían obstaculizar las decisiones de localización de individuos y empresas, un mercado de vivienda en alquiler débil que podría limitar las decisiones de inmigración laboral, o que regiones con mayor tasa de vivienda en propiedad conllevan tiempos de trayecto al trabajo más largos y, por tanto, mayores costes laborales. Sus resultados confirman los de Coulson y Fisher (2009), encontrándose para los estados de EEUU evidencia a nivel micro y macro de que, aun teniendo los propietarios de vivienda una probabilidad de empleo mayor, un aumento en la tasa de vivienda en propiedad del estado en que reside el individuo genera un posterior aumento de la tasa de desempleo en este estado. Laamanen (2013) llega a similares conclusiones con datos micro para Finlandia.

En resumen, las investigaciones previas que analizan el efecto de la forma de tenencia de la vivienda habitual sobre la situación de empleo del individuo, tanto a nivel teórico como empírico, no son concluyentes. Estos resultados contradictorios es lo que se ha venido a llamar en la literatura relacionada como el «puzzle de Oswald» (Van Ewijk y Van Leuvenjstein, 2009, pág. 3).

El presente trabajo aprovecha la variación temporal en términos de empleo y forma de tenencia de la vivienda generada por la reciente crisis económica en España para intentar aportar un poco de luz a este problema y poner así en cuestión los resultados precedentes. Con este objetivo, en la sección 2 presentamos las limitaciones de la evidencia agregada existente, tanto a nivel nacional como internacional, para pasar a estimar en la sección 3, basándonos en datos de panel de la Encuesta de Condiciones de Vida (ECV) de la Unión Europea correspondientes al periodo 2004-2013, diferentes modelos probit dinámicos univariantes y bivariantes que nos permitirán analizar la situación de empleo en España. La modelización econométrica propuesta facilitará afrontar distintos problemas identificados en la literatura previa y que no han sido abordados en su globalidad hasta ahora. En primer lugar, el uso de datos de panel micro permite incorporar el efecto de la heterogeneidad inobservada. En segundo lugar, consideramos explícitamente la endogeneidad de la forma de tenencia de la vivienda como variable explicativa de la situación de empleo, algo que preocupa especialmente en la literatura relacionada. Por último, el modelo probit dinámico utilizado permitirá deslindar la dependencia del estado espuria (esto es, aquella generada por la heterogeneidad inobservada), de la verdadera (es decir, tras controlar por la heterogeneidad inobservada, aquella causada porque la situación de empleo del individuo en un periodo altera significativamente la probabilidad de empleo en periodos subsiguientes). 
En la sección 4 se discuten los resultados obtenidos, mientras que la sección 5 sintetiza las principales conclusiones derivadas. Encontramos que, si abordamos los tres problemas empíricos mencionados, podemos afirmar que en el periodo estudiado los propietarios de vivienda en España, y especialmente los que tienen una hipoteca pendiente, están en una posición ligeramente mejor respecto de los inquilinos para afrontar los efectos de la crisis económica en términos de empleo. Adicionalmente, se evidencia que la tasa de vivienda en propiedad de la comunidad autónoma de residencia no genera ningún efecto significativo sobre la probabilidad de empleo de los individuos. Es importante destacar que si relajamos la atención de cualquiera de los problemas empíricos identificados, se llega a las mismas conclusiones (incorrectas en mi opinión) que Coulson y Fisher (2009), Blanchflower y Oswald (2013) o Laamanen (2013). Finalizamos con un apéndice con estadísticos descriptivos y detalles para las variables utilizadas.

\section{La evidencia agregada}

Si atendemos a la comparación internacional de la evolución de tasas de vivienda en propiedad y de tasas de desempleo realizada en los Gráficos 1 (a) y (b), respectivamente, observamos que España es un caso peculiar, con la tasa de vivienda en propiedad en promedio más alta de todos los países incluidos (en torno a 79,3 por 100 a lo largo del periodo) y una tasa de desempleo disparada desde el 2008 (junto con Grecia) hasta niveles en torno al 26 por 100 en 2013 y 24,5 por 100 en 2014, muy por encima de la media del resto de países considerados.

Confrontando ambas tasas en el Gráfico 1 (c), advertimos en la esquina inferior izquierda un grupo de países (Alemania, Francia, Reino Unido, Japón, EEUU) con las menores tasas de vivienda en propiedad, por debajo del 70 por 100 y con el caso extremo de Alemania con tasas de vivienda en propiedad en torno al 53 por 100, junto con unas tasas de desempleo que han aumentado en el periodo de crisis pero siempre manteniéndose bien por debajo del 10 por 100, con picos en Francia del 10,2 por 100 en 2013 y 2014. Por otro lado nos encontramos con un amplio abanico de países con una alta tasa de penetración de la vivienda en propiedad, por encima del 70 por 100, y un variado comportamiento del mercado laboral, yendo desde los casos de Portugal, Italia o Irlanda con tasas de paro tras el periodo de crisis en torno a 1114 por 100, y los casos extremos de España y Grecia que casi duplican estas tasas de paro en 2014. De este último gráfico cabe destacar las trayectorias casi verticales que han sostenido un buen número de países durante el periodo analizado, manteniendo casi constante la tasa de vivienda en propiedad, con un margen de $\pm 1,6$ puntos porcentuales, al tiempo que se ha disparado la tasa de paro como consecuencia de la crisis económica acontecida, de forma abrupta como es el caso de España, Grecia, Portugal o Italia, y también de manera más atemperada como Alemania, Francia o Japón. En contraste, los países del ámbito anglosajón como Irlanda, Reino Unido y EEUU, han respondido al periodo analizado con una «trayectoria hacia atrás», con 


\section{GRÁFICO 1}

\section{EVOLUCIÓN INTERNACIONAL DE LA TASA DE VIVIENDA EN PROPIEDAD Y TASA DE PARO PARA EL PERIODO 2004-14}

(a)

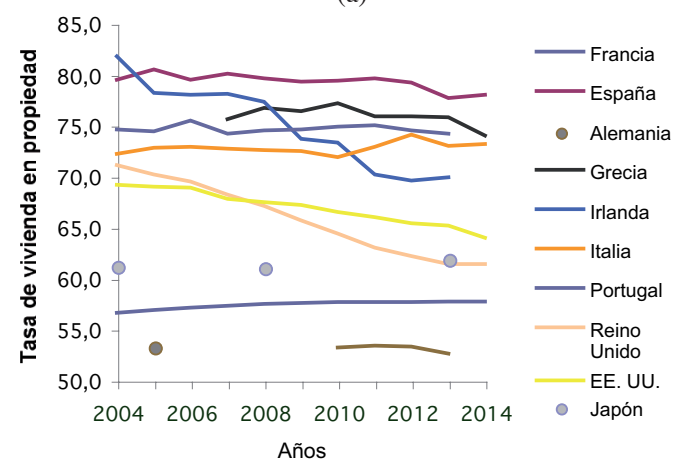

(b)

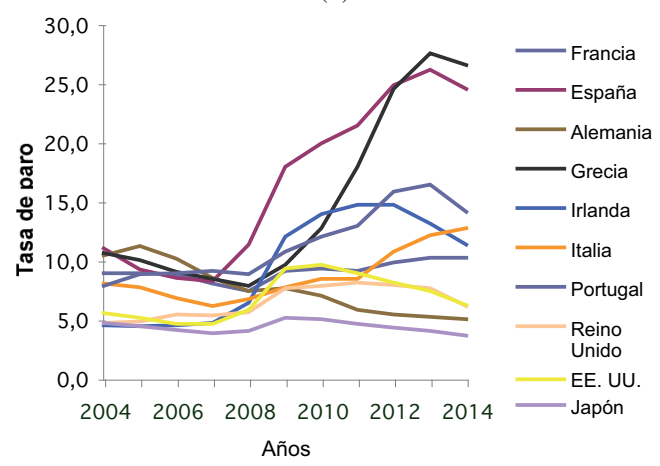

(c)

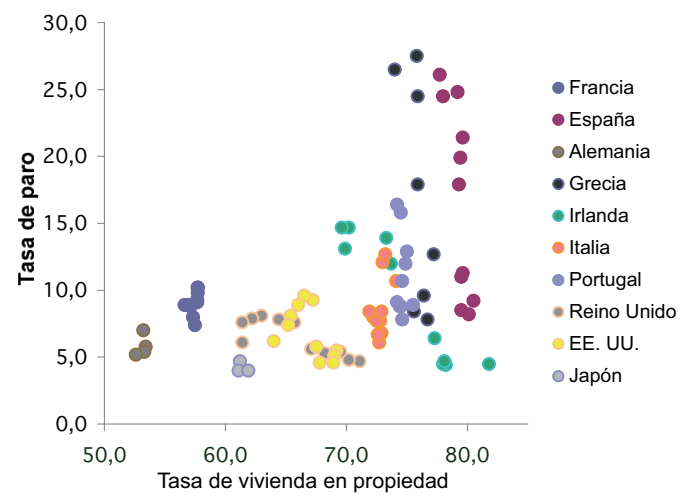

FUENTE: Elaboración propia y Eurostat e Institutos de Estadística nacionales. 
una marcada reducción de la tasa de vivienda en propiedad, de alrededor a 9,7, 11,9 y 5,2 puntos porcentuales, respectivamente, acompañada de un aumento de la tasa de desempleo de en torno a 4 puntos porcentuales en los años de mayor impacto de la crisis económica, excepción hecha de Irlanda que vio cómo se incrementaba su tasa de paro hasta en 10 puntos porcentuales en este periodo.

En consecuencia, se advierten diferencias en el comportamiento del mercado laboral a nivel internacional, independientemente de la cobertura de vivienda en propiedad que posea el stock de viviendas habituales nacional, a buen seguro debido principalmente, entre otros factores, a la diferente legislación laboral vigente en cada país, la composición sectorial de la economía, la balanza comercial, etc. Por tanto, para aislar mejor el posible efecto que ejerce la forma de tenencia de la vivienda habitual sobre la situación de empleo de los individuos cabe recurrir al ámbito nacional para excluir, de entrada, el efecto diferencial de la legislación laboral nacional. A este respecto, si acudimos a datos agregados de las CCAA. en España, vemos en el Gráfico 2 como una simple regresión agrupada indica que un incremento de un punto porcentual de la tasa de vivienda en propiedad provoca una caída estadísticamente significativa al 1 por 100 de probabilidad de en torno a 2,9 puntos porcentuales en la tasa de paro regional, resultado corroborado si incluimos variables dummies anuales para recoger la trayectoria temporal del periodo de crisis económica.

Sin embargo, si añadimos a la regresión anterior efectos individuales para las CCAA, el efecto de la tasa de vivienda en propiedad sobre la tasa de desempleo

\section{GRÁFICO 2}

\section{CONFRONTACIÓN DE LAS TASAS DE VIVIENDA EN PROPIEDAD Y DE PARO A NIVEL DE LAS COMUNIDADES AUTÓNOMAS EN ESPAÑA PARA EL PERIODO 2004-2014}

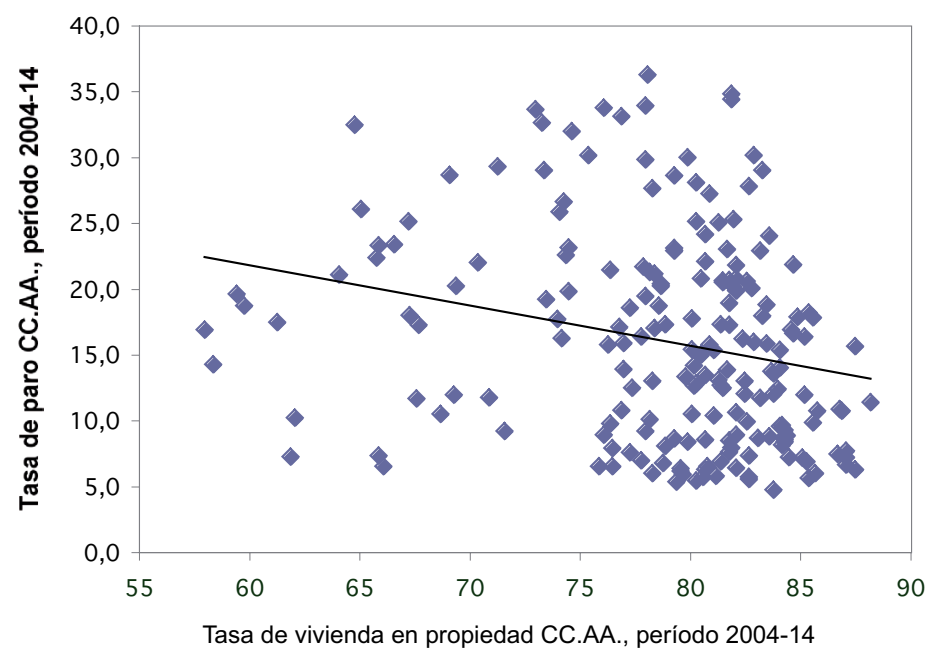

FUENTE: Elaboraciòn propia y EPA, ECV, INE. 
se difumina y deja de ser estadísticamente significativo, en contradicción con los resultados obtenidos por Barrios y Rodríguez (2004) con datos de corte transversal para las provincias españolas en el año 1991, específicamente que las provincias con un punto porcentual adicional en sus tasas de vivienda en propiedad conllevan tasas de desempleo 0,22 puntos porcentuales menores.

No obstante la evidencia agregada anterior hay que tomarla con suma cautela debido fundamentalmente a que hemos agrupado datos de corte transversal y a que no hemos tenido en cuenta la simultaneidad existente entre la situación de empleo y la decisión sobre la forma de tenencia de la vivienda habitual (causalidad inversa). Por ello, pasamos a continuación al análisis a nivel micro del fenómeno estudiado.

\section{Datos y modelización econométrica}

\subsection{Datos}

Empleamos los microdatos anuales longitudinales de la Encuesta de Condiciones de Vida europea (ECV. Fuente: INE) correspondientes a España para el periodo 2004-2013. El diseño de esta operación estadística es el de un panel rotante en el que cada hogar se sigue durante un periodo máximo de 4 años, renovándose un cuarto de la muestra anualmente. La ECV contiene información tanto a nivel de hogar como al nivel de los individuos que lo componen.

La muestra finalmente utilizada está constituida por aquellos responsables de la vivienda de residencia con edad entre 18 y 64 años (incluidos), que son económicamente activos, y no ocupan la vivienda por cesión gratuita o esta lo es de renta inferior a la de mercado ${ }^{1}$. También, debido a que procederemos a estimar modelos dinámicos con un retardo en las variables dependientes consideradas, se incluyen solo aquellos individuos observados al menos en dos periodos consecutivos y aquellos sin observaciones perdidas en cualquiera de las variables utilizadas. Como resultado de todo ello, tenemos un panel no balanceado de 15.169 individuos con un total de 42.085 observaciones anuales.

El Cuadro 1 recoge el tamaño de muestra final desglosado anualmente entre inquilinos a precio de mercado/propietarios de la vivienda y empleados/desempleados. Se puede observar que el porcentaje de inquilinos crece ligeramente desde un 8,9 por 100 en 2004 hasta un 10,7 por 100 en 2013, conllevando esto la correspondiente disminución en el porcentaje de propietarios desde un 91,8 por 100 hasta un 89,3 por 100 al final del periodo analizado. Si atendemos a la muestra total, es de destacar que los inquilinos

\footnotetext{
${ }^{1}$ El alquiler con renta inferior a la de mercado incluye el alquiler público, el alquiler a precio reducido por parte del empleador u otra organización, y el alquiler de renta antigua anterior al Decreto Boyer. Estos hogares son excluidos debido a que por sus peculiares características en cuanto a movilidad y empleo (véase, por ejemplo, Battu et al., 2008), su consideración podría distorsionar el objetivo fundamental del presente trabajo, esto es, evaluar el impacto de las dos formas de tenencia de vivienda principales (alquiler de mercado/ propiedad) sobre la situación de empleo del individuo.
} 
poseen una tasa de desempleo casi 8 puntos porcentuales superior a la de los propietarios. por el contrario, el profundo impacto de la crisis económica ha generado que las tasas de desempleo de ambos colectivos casi se hayan doblado en el mismo periodo, pasando de una media de 9,7 por 100 y 5,9 por 100 durante los años 2004-2008, respectivamente, para inquilinos y propietarios, hasta alcanzar unos valores respectivos de 24,6 por 100 y 14,9 por 100 en los años subsiguientes (2009-2013).

\section{CUADRO 1}

TAMAÑO DE LA MUESTRA

\begin{tabular}{|c|c|c|c|c|}
\hline & & $\begin{array}{l}\text { Porcentaje de } \\
\text { desempleados }\end{array}$ & $\begin{array}{l}\text { Porcentaje de } \\
\text { empleados }\end{array}$ & Total \\
\hline 2004 & $\begin{array}{l}\text { Inquilinos a precio de mercado } \\
\text { Propietarios de vivienda } \\
\text { Total }\end{array}$ & $\begin{array}{r}10,82 \\
6,62 \\
6,99\end{array}$ & $\begin{array}{l}89,18 \\
93,38 \\
93,01\end{array}$ & $\begin{array}{r}194 \\
1980 \\
2174\end{array}$ \\
\hline 2005 & $\begin{array}{l}\text { Inquilinos a precio de mercado } \\
\text { Propietarios de vivienda } \\
\text { Total }\end{array}$ & $\begin{array}{r}12,24 \\
5,22 \\
5,86 \\
\end{array}$ & $\begin{array}{l}87,76 \\
94,78 \\
94,14 \\
\end{array}$ & $\begin{array}{r}343 \\
3448 \\
3791 \\
\end{array}$ \\
\hline 2006 & $\begin{array}{l}\text { Inquilinos a precio de mercado } \\
\text { Propietarios de vivienda } \\
\text { Total }\end{array}$ & $\begin{array}{l}8,60 \\
5,10 \\
5,44\end{array}$ & $\begin{array}{l}91,40 \\
94,90 \\
94,56\end{array}$ & $\begin{array}{r}372 \\
3509 \\
3881\end{array}$ \\
\hline 2007 & $\begin{array}{l}\text { Inquilinos a precio de mercado } \\
\text { Propietarios de vivienda } \\
\text { Total }\end{array}$ & $\begin{array}{l}7,64 \\
5,48 \\
5,69\end{array}$ & $\begin{array}{l}92,36 \\
94,52 \\
94,31\end{array}$ & $\begin{array}{r}458 \\
4144 \\
4602\end{array}$ \\
\hline 2008 & $\begin{array}{l}\text { Inquilinos a precio de mercado } \\
\text { Propietarios de vivienda } \\
\text { Total }\end{array}$ & $\begin{array}{l}9,13 \\
6,90 \\
7,12 \\
\end{array}$ & $\begin{array}{l}90,87 \\
93,10 \\
92,88\end{array}$ & $\begin{array}{r}515 \\
4667 \\
5182 \\
\end{array}$ \\
\hline 2009 & $\begin{array}{l}\text { Inquilinos a precio de mercado } \\
\text { Propietarios de vivienda } \\
\text { Total }\end{array}$ & $\begin{array}{l}22,34 \\
11,33 \\
12,50\end{array}$ & $\begin{array}{l}77,66 \\
88,67 \\
87,50\end{array}$ & $\begin{array}{r}573 \\
4827 \\
5400\end{array}$ \\
\hline 2010 & $\begin{array}{l}\text { Inquilinos a precio de mercado } \\
\text { Propietarios de vivienda } \\
\text { Total }\end{array}$ & $\begin{array}{l}24,33 \\
12,87 \\
14,14\end{array}$ & $\begin{array}{l}75,67 \\
87,13 \\
85,86\end{array}$ & $\begin{array}{r}522 \\
4174 \\
4696\end{array}$ \\
\hline 2011 & $\begin{array}{l}\text { Inquilinos a precio de mercado } \\
\text { Propietarios de vivienda } \\
\text { Total }\end{array}$ & $\begin{array}{l}24,57 \\
13,36 \\
14,59\end{array}$ & $\begin{array}{l}75,43 \\
86,64 \\
85,41\end{array}$ & $\begin{array}{r}464 \\
3751 \\
4.215\end{array}$ \\
\hline 2012 & $\begin{array}{l}\text { Inquilinos a precio de mercado } \\
\text { Propietarios de vivienda } \\
\text { Total }\end{array}$ & $\begin{array}{l}25,83 \\
17,51 \\
18,40\end{array}$ & $\begin{array}{l}74,17 \\
82,49 \\
81,60\end{array}$ & $\begin{array}{r}515 \\
4301 \\
4.816\end{array}$ \\
\hline 2013 & $\begin{array}{l}\text { Inquilinos a precio de mercado } \\
\text { Propietarios de vivienda } \\
\text { Total }\end{array}$ & $\begin{array}{l}26,12 \\
19,41 \\
20,13 \\
\end{array}$ & $\begin{array}{l}73,88 \\
80,59 \\
79,87 \\
\end{array}$ & $\begin{array}{r}515 \\
2.972 \\
3.328 \\
\end{array}$ \\
\hline Total & $\begin{array}{l}\text { Inquilinos a precio de mercado } \\
\text { Propietarios de vivienda } \\
\text { Total }\end{array}$ & $\begin{array}{l}17,90 \\
10,47 \\
11,23\end{array}$ & $\begin{array}{l}82,10 \\
89,53 \\
88,77\end{array}$ & $\begin{array}{r}4.312 \\
37.773 \\
42.085\end{array}$ \\
\hline
\end{tabular}


En el Cuadro 2 presentamos las tasas de transición bivariantes anuales para analizar precisamente la dinámica de empleo y forma de tenencia de la vivienda de la muestra estudiada. Se observa una relativa persistencia a lo largo todo el periodo analizado tanto del porcentaje de inquilinos empleados como de los propietarios empleados, con unas medias respectivas de 83 por 100 y 95 por 100 de individuos en estos grupos que permanecen en la misma situación el año siguiente. Al mismo tiempo, nuevamente como consecuencia de la crisis económica, las transiciones de una situación de empleo a una de desempleo aumentan de unas medias respectivas para inquilinos y propietarios de 5,6 por 100 y 2,7 por 100 en el periodo 2004-2008, hasta unas medias de 12,8 por 100 y 6,2 por 100, respectivamente, en el periodo 2009-2013. Adviértase que las tasas de transición de empleo a desempleo para los inquilinos doblan aproximadamente a las correspondientes de propietarios a lo largo del periodo completo. Paralelamente, la probabilidad de acceder a un empleo en el siguiente periodo para un inquilino o un propietario desempleados en el año anterior decrece marcadamente después de 2008, de unas medias respectivas entre 2004-2008 de 57,2 por 100 y 45,7 por 100, hasta situarse en torno a 32,6 por 100 y 27,6 por 100, respectivamente, a lo largo del periodo 2009-2013.

\section{CUADRO 2}

TASAS DE TRANSICIÓN BIVARIANTES ANUALES DE EMPLEO Y FORMA DE TENENCIA DE LA VIVIENDA

\begin{tabular}{|c|c|c|c|c|c|}
\hline \multicolumn{6}{|c|}{$2004 / 05$} \\
\hline & IE & ID & PE & PD & Observaciones \\
\hline Inquilinos empleados (IE) & 82,1 & 6,4 & 10,4 & 1,2 & 173 \\
\hline Inquilinos desempleados (ID) & 42,9 & 57,1 & 0,0 & 0,0 & 21 \\
\hline Propietarios empleados (PE) & 0,3 & 0,0 & 97,1 & 2,5 & 1.849 \\
\hline Propietarios desempleados (PD) & 1,5 & 0,0 & 51,1 & 47,3 & 131 \\
\hline Total & 7,3 & 1,1 & 86,5 & 5,1 & 2.174 \\
\hline \multicolumn{6}{|c|}{ 2005/06 } \\
\hline & IE & ID & $\mathbf{P E}$ & PD & Observaciones \\
\hline Inquilinos empleados (IE) & 81,6 & 4,2 & 14,2 & 0,0 & 190 \\
\hline Inquilinos desempleados (ID) & 61,5 & 26,9 & 11,5 & 0,0 & 26 \\
\hline Propietarios empleados (PE) & 0,4 & 0,0 & 96,7 & 2,9 & 2.028 \\
\hline Propietarios desempleados (PD) & 1,0 & 0,0 & 51,5 & 47,4 & 97 \\
\hline Total & 7,7 & 0,6 & 87,2 & 4,5 & 2.341 \\
\hline \multicolumn{6}{|c|}{ 2006/07 } \\
\hline & IE & ID & PE & PD & Observaciones \\
\hline Inquilinos empleados (IE) & 85,6 & 5,2 & 8,0 & 1,2 & 250 \\
\hline Inquilinos desempleados (ID) & 65,2 & 34,8 & 0,0 & 0,0 & 23 \\
\hline Propietarios empleados (PE) & 0,4 & 0,0 & 97,4 & 2,2 & 2.400 \\
\hline Propietarios desempleados (PD) & 0,0 & 0,0 & 45,2 & 54,8 & 115 \\
\hline Total & 8,5 & 0,8 & 86,4 & 4,3 & 2.788 \\
\hline
\end{tabular}


CUADRO 2 (continuación)

TASAS DE TRANSICIÓN BIVARIANTES ANUALES DE EMPLEO Y FORMA DE TENENCIA DE LA VIVIENDA*

\begin{tabular}{|c|c|c|c|c|c|}
\hline \multicolumn{6}{|c|}{$2007 / 08$} \\
\hline & IE & ID & PE & PD & Observaciones \\
\hline Inquilinos empleados (IE) & 87,1 & 6,5 & 6,1 & 0,3 & 310 \\
\hline Inquilinos desempleados (ID) & 59,1 & 31,8 & 4,5 & 4,5 & 22 \\
\hline Propietarios empleados (PE) & 0,3 & 0,0 & 96,4 & 3,2 & 2.909 \\
\hline Propietarios desempleados (PD) & 0,0 & 0,6 & 34,7 & 64,7 & 170 \\
\hline Total & 8,6 & 0,9 & 84,5 & 6,0 & 3.411 \\
\hline \multicolumn{6}{|c|}{$2008 / 09$} \\
\hline & IE & ID & PE & PD & Observaciones \\
\hline Inquilinos empleados (IE) & 79,8 & 15,3 & 4,9 & 0,0 & 326 \\
\hline Inquilinos desempleados (ID) & 24,2 & 72,7 & 0,0 & 3,0 & 33 \\
\hline Propietarios empleados (PE) & 0,4 & 0,0 & 93,9 & 5,6 & 3.095 \\
\hline Propietarios desempleados (PD) & 0,4 & 0,4 & 24,9 & 74,3 & 237 \\
\hline Total & 7,6 & 2,1 & 80,8 & 9,5 & 3.691 \\
\hline \multicolumn{6}{|c|}{$2009 / 10$} \\
\hline & IE & ID & PE & PD & Observaciones \\
\hline Inquilinos empleados (IE) & 83,5 & 10,1 & 5,7 & 0,6 & 316 \\
\hline Inquilinos desempleados (ID) & 28,9 & 67,5 & 1,2 & 2,4 & 83 \\
\hline Propietarios empleados (PE) & 0,4 & 0,0 & 94,4 & 5,2 & 3.057 \\
\hline Propietarios desempleados (PD) & 0,5 & 0,3 & 34,4 & 64,8 & 381 \\
\hline Total & 7,8 & 2,3 & 79,2 & 10,7 & 3.837 \\
\hline \multicolumn{6}{|c|}{$2010 / 11$} \\
\hline & IE & ID & PE & PD & Observaciones \\
\hline Inquilinos empleados (IE) & 82,7 & 11,8 & 5,1 & 0,4 & 237 \\
\hline Inquilinos desempleados (ID) & 40,9 & 56,8 & 1,1 & 1,1 & 88 \\
\hline Propietarios empleados (PE) & 0,2 & 0,0 & 94,6 & 5,1 & 2.294 \\
\hline Propietarios desempleados (PD) & 0,3 & 0,3 & 32,6 & 66,8 & 316 \\
\hline Total & 8,1 & 2,7 & 77,9 & 11,3 & 2.935 \\
\hline \multicolumn{6}{|c|}{$2011 / 12$} \\
\hline & IE & ID & $\mathbf{P E}$ & PD & Observaciones \\
\hline Inquilinos empleados (IE) & 82,6 & 12,6 & 3,7 & 1,1 & 190 \\
\hline Inquilinos desempleados (ID) & 34,0 & 64,0 & 0,0 & 2,0 & 50 \\
\hline Propietarios empleados (PE) & 0,3 & 0,1 & 92,0 & 7,7 & 1.893 \\
\hline Propietarios desempleados (PD) & 0,4 & 0,7 & 22,7 & 76,3 & 278 \\
\hline Total & 7,5 & 2,4 & 75,1 & 15,0 & 2.411 \\
\hline \multicolumn{6}{|c|}{$2012 / 13$} \\
\hline & IE & ID & PE & PD & Observaciones \\
\hline Inquilinos empleados (IE) & 82,4 & 13,9 & 3,4 & 0,4 & 267 \\
\hline Inquilinos desempleados (ID) & 34,9 & 62,8 & 1,2 & 1,2 & 86 \\
\hline Propietarios empleados (PE) & 0,5 & 0,1 & 92,0 & 7,4 & 2.461 \\
\hline Propietarios desempleados (PD) & 0,2 & 0,0 & 23,5 & 76,3 & 514 \\
\hline Total & 7,9 & 2,8 & 72,0 & 17,3 & 3.328 \\
\hline
\end{tabular}

NOTA: * Tasas de transición en porcentaje calculadas para aquellos individuos que permanecen en la muestra al menos en cada uno de los dos años consecutivos recogidos. 


\subsection{Variables utilizadas}

En la ecuación de tenencia de vivienda se considera la variable dependiente binaria TENEN que toma el valor 1 si la vivienda habitual se ocupa en propiedad al tiempo de realizar la encuesta, y 0 si se ocupa en alquiler. La variable dependiente en la ecuación de empleo será la binaria EMPLEO que indicará si el responsable de la vivienda está empleado (1) o desempleado (0) cuando se le realizó la encuesta.

En ambas ecuaciones, controlamos por la edad, el género, el nivel educativo, si vive en pareja (con o sin base legal), el número de miembros del hogar, los años de experiencia laboral, el estado de salud, el nacimiento en España, la densidad poblacional del área local de residencia, las tasas de vivienda en propiedad y de desempleo en la comunidad autónoma de residencia, así como variables indicadoras del año en que se realiza la observación. Adicionalmente, siguiendo a Coulson y Fisher (2009), para ayudar a identificar el efecto de la forma de tenencia de la vivienda habitual sobre el empleo, incluimos en la ecuación de tenencia de vivienda el porcentaje de hogares en viviendas unifamiliares aisladas o adosadas en la comunidad autónoma de residencia. Asumimos que esta variable no está correlacionada con la situación de empleo del individuo para utilizarla como variable instrumental. Recogemos los estadísticos descriptivos de las variables dependientes y de control consideradas en un apéndice final, junto a los detalles sobre sus definiciones. De la observación de estos estadísticos destacamos las diferencias existentes entre la distribución de las variables incluidas para el colectivo de propietarios frente al de inquilinos, como se puede deducir a través de la significatividad mostrada por la mayoría de tests t para la diferencia de medias entre ambos grupos. Precisamente por esta razón resulta importante controlar por las divergencias observadas para evitar que el sesgo de selección provoque distorsiones a la hora de estimar los efectos de la forma de tenencia de la vivienda sobre el indicador de empleo.

\subsection{Modelización econométrica}

Se plantea un modelo econométrico que permita abordar tres problemas fundamentales que nos encontramos al estudiar el fenómeno que nos ocupa. En primer lugar, la utilización de datos de panel permite aislar el efecto sobre la situación de empleo de los individuos tanto de la heterogeneidad observada, derivada de las variables de control, como de la inobservada. En segundo término, tenemos en cuenta la endogeneidad de la elección de tenencia de la vivienda como variable explicativa de la situación de empleo mediante la modelización conjunta de la forma de tenencia de la vivienda y del estado de empleo, considerando que los factores inobservados que afectan a ambas situaciones pueden estar correlacionados. Por último, la persistencia en el tiempo de la situación de empleo, evidenciada en el Cuadro 2, apunta a la necesidad de incorporar técnicas que nos permitan deslindar la dependencia del estado espuria, provocada por la heterogeneidad inobservada, de la verdadera, esto 
es, aquella generada porque la situación de empleo del individuo en un periodo altera significativamente la probabilidad de empleo en periodos subsiguientes. La implementación de un modelo probit bivariante dinámico con efectos aleatorios similar al utilizado en Alessie et al. (2004), Stewart (2007) o Picchio y Van Ours (2013), nos permite afrontar este último aspecto. Aquí es importante señalar que, de no tenerse en cuenta explícitamente esta dependencia del estado verdadera, ésta podría ser confundida con el efecto de la forma de tenencia de la vivienda, que como vimos en el Cuadro 2 también es una decisión muy persistente en el tiempo, provocando una sobreestimación de este último.

En consecuencia, modelizamos conjuntamente la elección de tenencia de vivienda y la situación de empleo mediante un modelo probit bivariante dinámico con efectos aleatorios correlacionados con las variables de control, siguiendo a Mundlak (1978) y Chamberlain (1980), y cuyo problema de condiciones iniciales, esto es, que la tenencia de vivienda y la situación de empleo observadas inicialmente para cada individuo pueda depender de la heterogeneidad inobservada, se afronta mediante la técnica de Wooldridge (2005). Este modelo vendrá dado por las ecuaciones:

$$
\begin{aligned}
& E_{i t}=1\left(x_{1 i t}^{\prime} \beta_{1}+\gamma_{11} E_{i t-1}+\gamma_{12} H_{i t-1}+\bar{x}_{1 i t}^{\prime} \delta_{1}+\lambda_{11} E_{i 0}+\lambda_{12} E_{i 0}+a_{1 i}+\varepsilon_{1 i t}\right) \\
& H_{i t}=1\left(x_{2 i t}^{\prime} \beta_{2}+\gamma_{21} E_{i t-1}+\gamma_{22} H_{i t-1}+\bar{x}_{2 i t}^{\prime} \delta_{2}+\lambda_{21} E_{i 0}+\lambda_{22} H_{i 0}+a_{2 i}+\varepsilon_{2 i t}\right)
\end{aligned}
$$

donde 1 representa la función indicador, $i=1, \ldots, N$ se refiere a los individuos y $t=1, \ldots, T$ al periodo de tiempo (años); $E_{i t}$ y $H_{i t}$ son, respectivamente, las variables binarias de EMPLEO y TENEN; $x_{1 i t}$ y $x_{2 i t}$ son vectores de variables de control estrictamente exógenas que explican, respectivamente, la situación de empleo y la forma de tenencia de la vivienda; $\bar{x}_{1 i t}^{\prime} y \bar{x}_{2 i t}^{\prime}$ son los vectores de medias individuales de aquellas variables en $x_{1 i t}$ y $x_{2 i t}$, respectivamente, que varían a través del tiempo; $E_{i 0} \mathrm{y}$ $H_{i 0}$ son la primera observación para cada individuo de su estado de empleo y de su forma de tenencia de la vivienda habitual; $\beta_{1}, \beta_{2}, \delta_{1}, \delta_{2}$ son vectores de parámetros conformes, $\gamma_{11}, \gamma_{12}, \gamma_{21}, \gamma_{22}, \lambda_{11}, \lambda_{12}, \lambda_{21}, \lambda_{22}$ son parámetros simples; $a_{1 i}$ y $a_{2 i}$ sintetizan la heterogeneidad individual inobservada restante que se supone independiente de las variables de control observadas y que sigue una distribución normal bivariante de media cero y varianzas respectivas $\sigma_{a_{1}}^{2}$ y $\sigma_{a_{2}}^{2}$ y covarianza $\rho_{a}$; y finalmente, $\varepsilon_{1 i t}$ and $\varepsilon_{2 i t}$ son errores independientes a través del tiempo y también respecto de $a_{1 i}, \mathrm{y}_{2 i}$, y con distribución normal bivariante estándar con covarianza $\rho$.

Adviértase que [1] puede estimarse por máxima verosimilitud condicionada como un modelo probit bivariante con efectos aleatorios. Como señalan Alessie et al. (2004) o Stewart (2007), algunos casos particulares del modelo [1] son especialmente relevantes. Si $\gamma_{12}=0$, la ecuación de empleo en [1] no depende de la tenencia de vivienda retardada y en consecuencia no existe efecto micro de ésta última sobre la situación de empleo. Si $\gamma_{12} \neq 0$, pero $\rho_{a}=\rho=0$, entonces $H_{i t-1}$ es débilmente exógena en la ecuación de empleo en [1], y los parámetros de esta ecuación pueden ser estimados consistentemente por separado mediante el estimador 
de Wooldridge (2005) como un modelo probit dinámico univariante con efectos aleatorios correlacionados. Por último, si $\gamma_{12} \neq 0$ y $\rho_{a} \neq 0$ o $\rho \neq 0$, nos encontramos ante el caso de correlación cruzada en el que se requiere una estimación conjunta del modelo bivariante [1].

A continuación, estimamos consistentemente los efectos parciales medios (EPM) sobre el empleo de la forma de tenencia de la vivienda retardada $\left(\pi_{1}\right)$, de la situación de empleo retardada $\left(\pi_{2}\right)$, la cual nos indica la importancia de la persistencia del estado de empleo, y de la tasa de vivienda en propiedad de la CCAA de residencia $\left(\pi_{3}\right)$, siguiendo a Wooldridge (2005). Para ello, calculamos:

$$
\frac{1}{N} \sum_{i=1}^{N} \Phi\left(x_{1 i t}^{\prime} \hat{\beta}_{a_{1} 1}+\hat{\gamma}_{a_{1} 11} E_{i t-1}+\hat{\gamma}_{a_{1} 12} H_{i t-1}+\bar{x}_{1 i t}^{\prime} \hat{\delta}_{a_{1} 1}+\hat{\lambda}_{a_{1} 11} E_{i 0}+\hat{\lambda}_{a_{1} 12} H_{i 0}\right)
$$

donde $\Phi$ es la función de distribución normal estándar y el subíndice $a_{1}$ denota los coeficientes estimados para el modelo [1] divididos por $\sqrt{1+\hat{\sigma}_{a_{1}}^{2}}$, con $\hat{\sigma}_{a_{1}}^{2}$ la varianza estimada de $a_{1}$. Tomaremos diferencias en [2] con, respectivamente, $H_{i t-1}=1 \mathrm{y}$ $H_{i t-1}=0$ para $\pi_{1}, E_{i t-1}=1$ y $E_{i t-1}=0$ para $\pi_{2}$, y calcularemos la derivada de [2] con respecto a la tasa de vivienda en propiedad regional para $\pi_{3}$, y posteriormente calcularemos la media a lo largo de todo el periodo estudiado para obtener los EPM en todo este periodo, o a lo largo de una año determinado para calcular los EPM anuales. Los correspondientes errores estándar se obtienen mediante el método delta.

El modelo utilizado permite resolver el problema de identificación del efecto causal de la forma de tenencia de la vivienda sobre la situación de empleo del individuo de varias maneras. Primero, su estructura recursiva posibilita considerar la elección de tenencia de vivienda hecha un año antes como predeterminada con respecto a la situación de empleo del año posterior. También, el periodo de crisis económica analizado proporcionará variabilidad exógena en ambas variables dependientes a lo largo del mismo, lo cual, unido con la estabilidad en el tiempo asumida para los parámetros estructurales del modelo, facilitará la identificación. No obstante, podría argumentarse que el capital inicial preciso para la adquisición de una vivienda, así como el frecuente recurso a los mercados financieros para materializar una hipoteca, podrían limitar el acceso a una vivienda en propiedad solo a aquellos individuos con un empleo estable y, por tanto, que la forma de tenencia de vivienda en un periodo previo podría estar correlacionada con la situación de empleo posterior. Por ello, para ayudar adicionalmente a la identificación, consideramos, análogamente a Coulson y Fisher (2009), como una variable instrumental en la ecuación de tenencia de vivienda en [1] la tasa de hogares cuya vivienda habitual es unifamiliar aislada o adosada en la comunidad autónoma de residencia (TUNIFAM), la cual es excluida de la ecuación de empleo. 


\section{Resultados}

El Cuadro 3 recoge los EPMs de interés a lo largo de todo el periodo estudiado bajo cuatro especificaciones diferentes a efectos comparativos. En la especificación 1 se considera un modelo probit dinámico agrupado univariante para el empleo en el que se supone que no hay heterogeneidad inobservada y que incluye la forma de tenencia de vivienda en el periodo anterior como variable explicativa que se asume exógena. La especificación 2 también es un modelo probit dinámico univariante para el empleo pero ahora con efectos aleatorios correlacionados, esto es, se admite la existencia de heterogeneidad inobservada, y de nuevo incluye la forma de tenencia de vivienda retardada como variable explicativa que permanece exógena. La especificación 3 es el modelo probit bivariante con efectos aleatorios correlacionados [1], en el que se mantiene la existencia de heterogeneidad inobservada y una elección de tenencia de vivienda endógena. En todas estas tres especificaciones se pierde un periodo temporal a causa de la dinámica del modelo, dando lugar a un panel no balanceado de 15.169 individuos con un total de 26.916 observaciones.

Finalmente, para analizar la sensibilidad de los resultados obtenidos ante las hipótesis distribucionales realizadas, en la especificación 4 se estima un modelo de panel lineal dinámico con efectos aleatorios utilizando el estimador GMM propuesto por Blundell y Bond (1998) aplicado a la muestra reducida que se obtiene para aquellos individuos observados al menos durante tres años consecutivos, una restricción debida a la naturaleza del estimador, y cuyos resultados son cualitativamente similares a los del estimador de Arellano y Bond (1991), pero más adecuados cuando $T$ es pequeño y la persistencia es alta. Ahora se utilizan como instrumentos los retardos superiores de la forma de tenencia de la vivienda, de la situación de empleo y de la tasa de vivienda en propiedad regional. Los detalles de los coeficientes estimados para todas

\section{CUADRO 3}

PRINCIPALES EFECTOS PARCIALES MEDIOS SOBRE EL EMPLEO

\begin{tabular}{|c|c|c|c|c|}
\hline & Especificación 1 & Especificación 2 & Especificación 3 & Especificación 4 \\
\hline$\pi_{1}$ & $\begin{array}{c}0,017 \\
(0,006)^{* *}\end{array}$ & $\begin{array}{c}0,017 \\
(0,006)^{* *}\end{array}$ & $\begin{array}{c}0,017 \\
(0,005)^{* *}\end{array}$ & $\begin{array}{c}0,342 \\
(0,237)\end{array}$ \\
\hline$\pi_{2}$ & $\begin{array}{c}0,501 \\
(0,011)^{* *}\end{array}$ & $\begin{array}{c}0,090 \\
(0,016)^{* *}\end{array}$ & $\begin{array}{c}0,092 \\
(0,008)^{* *}\end{array}$ & $\begin{array}{c}0,373 \\
(0,043)^{* *}\end{array}$ \\
\hline$\pi_{3}$ & $\begin{array}{c}-0,087 \\
(0,031)^{* *}\end{array}$ & $\begin{array}{c}-0,053 \\
(0,122)\end{array}$ & $\begin{array}{c}-0,052 \\
(0,111)\end{array}$ & $\begin{array}{c}-1,992 \\
(1,483)\end{array}$ \\
\hline N.o individuos & 15.169 & 15.169 & 15.169 & 7631 \\
\hline N.o observaciones & 26.916 & 26.916 & 26.916 & 27009 \\
\hline Log-verosimilitud & $-6.240,90$ & $-6.127,55$ & $-7.296,99$ & - \\
\hline
\end{tabular}

NOTA: Errores estándar entre paréntesis. * Significativo al 5 por 100, ** Significativo al 1 por $100 . \pi_{1}, \pi_{2}$ y $\pi_{3}$ son los EPMs sobre la probabilidad de empleo de, respectivamente, la forma de tenencia de vivienda retardada, la situación de empleo retardado, y la tasa de vivienda en propiedad en la CCAA de residencia. 
las especificaciones se omiten por las limitaciones de espacio y pueden ser facilitados bajo petición.

En todos los casos, como era de esperar, se corrobora que la situación de empleo previa influye significativamente al 1 por 100 de probabilidad sobre el estado de empleo en el periodo subsiguiente, validando se la especificación dinámica propuesta. Adicionalmente, un test $t$ sobre $\sigma_{a}^{2}$ o un test de ratio de verosimilitudes rechaza la especificación 1 frente a la especificación 2, confirmándose la presencia de efectos aleatorios correlacionados. Por otra parte, un test $t$ sobre la correlación de la heterogeneidad inobservada en ambas ecuaciones de [1], $\rho_{a}$, o sobre la covarianza de los errores en las mismas, $\rho$, no permite rechazar que sean nulas, y por tanto la especificación univariante 2 estima consistentemente los parámetros en la ecuación de empleo, como se puede observer en los valores similares obtenidos para los EPMs en las especificaciones 2 y 3 . La validez de la variable instrumental utilizada (TUNIFAM) se confirma puesto que un test $t$ y un test de ratio de verosimilitudes corrobora que es significativa al 1 por 100 de probabilidad en la ecuación de tenencia de vivienda, al tiempo que de nuevo un test $t$ y un test de ratio de verosimilitudes rechaza su inclusión en la ecuacion de empleo al mismo nivel de significatividad.

Comparando ahora los EPMs recogidos en el Cuadro 3 para la especificación 1 con los correspondientes para las especificaciones 2 y 3 , se observa que la mayor parte de la persistencia inicial en el empleo estimada bajo la especificación 1 se explica por la heterogeneidad inobservada incluida en las especificaciones 2 y 3 . En todo caso, la persistencia de la situación de empleo es significativa al nivel del 1 por 100, y considerando la heterogeneidad inobservada (especificaciones 2 y 3), la probabilidad de estar empleado en un determinado periodo aumenta en torno a 9 puntos porcentuales si el individuo estuvo empleado en el periodo inmediatamente anterior. Por otra parte, ser propietario de la vivienda en el periodo anterior genera un aumento de la probabilidad de empleo en el periodo subsiguiente que se mantiene estable, significativo al 1 por 100, y en torno a 1,7 puntos porcentuales en promedio en las tres especificaciones. Este resultado pone en entredicho una vez más la hipótesis de Oswald. Además, el efecto de la tasa regional de vivienda en propiedad sobre la probabilidad de empleo, inicialmente negativo y significativo al 1 por 100 de probabilidad, también se desvanece cuando tenemos en cuenta la heterogeneidad inobservada, en contra de los postulados de Blanchflower y Oswald (2013) o Laamanen (2013), cuestionándose por tanto la existencia de efectos indirectos sobre el empleo de la tasa de vivienda en propiedad regional. A este respecto, debemos señalar que ninguno de estos dos últimos trabajos considera explícitamente la naturaleza dinámica de la situación de empleo, ni incluyen en la modelización la heterogeneidad inobservada, a pesar de utilizar datos de panel en ambos casos.

Por último, el estimador lineal GMM de Blundell-Bond (1998) que utilizamos en la especificación 4, el cual no está sujeto a los supuestos distribucionales anteriores, apunta a una mayor persistencia en el empleo significativa, más en consonancia con el modelo probit agrupado de la especificación 1, así como a unos efectos estimados para la forma de tenencia de la vivienda previa y de la tasa de vivienda en propiedad 


\section{CUADRO 4}

\section{COMPROBACIÓN DE LA ROBUSTEZ DE LA ESPECIFICACIÓN 3 EN EL CUADRO 3}

\begin{tabular}{|c|c|c|c|c|c|c|}
\hline & $\begin{array}{c}\text { Sin } \\
\text { TPARO }\end{array}$ & $\begin{array}{c}\text { Con TPDAD } \\
(\boldsymbol{t}-\mathbf{1})\end{array}$ & $\begin{array}{c}\text { Con TPDAD } \\
(\boldsymbol{t}-\mathbf{2})\end{array}$ & $\begin{array}{c}\text { Con TPDAD } \\
(\boldsymbol{t}-\mathbf{3})\end{array}$ & $\begin{array}{c}\text { Con TPDAD } \\
(\boldsymbol{t}-\mathbf{4})\end{array}$ & $\begin{array}{c}\text { Con TPDAD } \\
(\boldsymbol{t} \text { - 5) }\end{array}$ \\
\hline$\pi_{1}$ & $\begin{array}{c}0,015 \\
(0,005)^{* *}\end{array}$ & $\begin{array}{c}0,013 \\
(0,005)^{* *}\end{array}$ & $\begin{array}{c}0,015 \\
(0,005)^{* *}\end{array}$ & $\begin{array}{c}0,011 \\
(0,005)^{*}\end{array}$ & $\begin{array}{c}0,012 \\
(0,005)^{*}\end{array}$ & $\begin{array}{c}0,011 \\
(0,007)\end{array}$ \\
\hline$\pi_{2}$ & $\begin{array}{c}0,097 \\
(0,009)^{* *}\end{array}$ & $\begin{array}{c}0,094 \\
(0,009)^{* *}\end{array}$ & $\begin{array}{c}0,103 \\
(0,009)^{* *}\end{array}$ & $\begin{array}{c}0,107 \\
(0,011)^{* *}\end{array}$ & $\begin{array}{c}0,119 \\
(0,013)^{* *}\end{array}$ & $\begin{array}{c}0,107 \\
(0,014)^{* *}\end{array}$ \\
\hline$\pi_{3}$ & $\begin{array}{c}-0,065 \\
(0,112)\end{array}$ & $\begin{array}{c}-0,056 \\
(0,112)\end{array}$ & $\begin{array}{c}-0,147 \\
(0,122)\end{array}$ & $\begin{array}{c}0,090 \\
(0,110)\end{array}$ & $\begin{array}{c}0,029 \\
(0,134)\end{array}$ & $\begin{array}{c}-0,095 \\
(0,205)\end{array}$ \\
\hline N. ${ }^{\circ}$ individuos & 15.169 & 13.719 & 12.626 & 11.435 & 9.944 & 8.381 \\
\hline N. ${ }^{\circ}$ observaciones & 26.916 & 24.742 & 22.401 & 19.613 & 16.202 & 12.511 \\
\hline Periodo incluido & $2004-13$ & $2005-13$ & $2006-13$ & $2007-13$ & $2008-13$ & $2009-13$ \\
\hline
\end{tabular}

NOTA: Errores estándar entre paréntesis. * Significativo al 5 por 100, ** Significativo al 1 por 100. $\pi_{1}, \pi_{2}$ y $\pi_{3}$ son los EPMs sobre la probabilidad de empleo de, respectivamente, la forma de tenencia de vivienda retardada, la situación de empleo retardado, y la tasa de vivienda en propiedad en la CCAA de residencia.

regional no significativamente diferentes de cero. No obstante, debemos tomar con cautela los resultados obtenidos con este último modelo puesto que, debido a la naturaleza del estimador, la muestra considerada se reduce casi en un 50 por 100, y también porque las características del panel de datos aquí utilizado no permiten contrastar la validez del modelo mediante un test sobre la correlación de segundo orden en los residuos, tal y como recomiendan Arelllano y Bond (1991).

En el Cuadro 4 se analiza a continuación la robustez de los resultados obtenidos anteriormente en el Cuadro 3 mediante la especificación 3 respecto de algunos aspectos relevantes. En primer lugar, cabría argumentar que puesto que se incluye la tasa de desempleo regional como una variable de control, ésta podría estar capturando una parte importante de la variación del empleo a nivel regional, y por tanto que reste poca variación para ser explicada por la tasa de vivienda en propiedad regional. Por ello, comenzamos estimando de nuevo la especificación 3 pero excluyendo la tasa de desempleo regional. Seguidamente, teniendo en cuenta que Blanchflower y Oswald (2013) postulan que el efecto de la tasa de vivienda en propiedad puede llevar un periodo de retardo largo, incluso de entre 4 y 5 años, estimamos de nuevo la especificación 3 utilizando ahora cualquier retardo hasta 5 años de la tasa de vivienda en propiedad regional, en lugar de sus valores contemporáneos ${ }^{2}$. En todos los casos, los resultados permanecen consistentes con los obtenidos en el Cuadro 3. También, si realizamos el mismo ejercicio con las especificaciones 2 y 4 , llegamos a resultados similares a los recogidos en el cuadro 3.

${ }^{2} \mathrm{Al}$ incorporar los retardos sucesivos de la tasa de vivienda en propiedad regional obtenida a partir de la ECV, la muestra utilizada en estas especificaciones se reduce al tenerse que eliminar consecutivamente las observaciones realizadas durante los primeros cinco años. 


\section{GRÁFICO 3}

EVOLUCIÓN ANUAL DE LOS TRES EPMS DE INTERÉS $\left(\pi_{1}, \pi_{2}, \pi_{3}\right)$ ESTIMADOS BAJO LA ESPECIFICACIÓN 3 (MEDIAS ANUALES), JUNTO CON INTERVALOS DEL 95 POR 100 DE CONFIANZA PARA LOS VALORES ANUALES ESTIMADOS
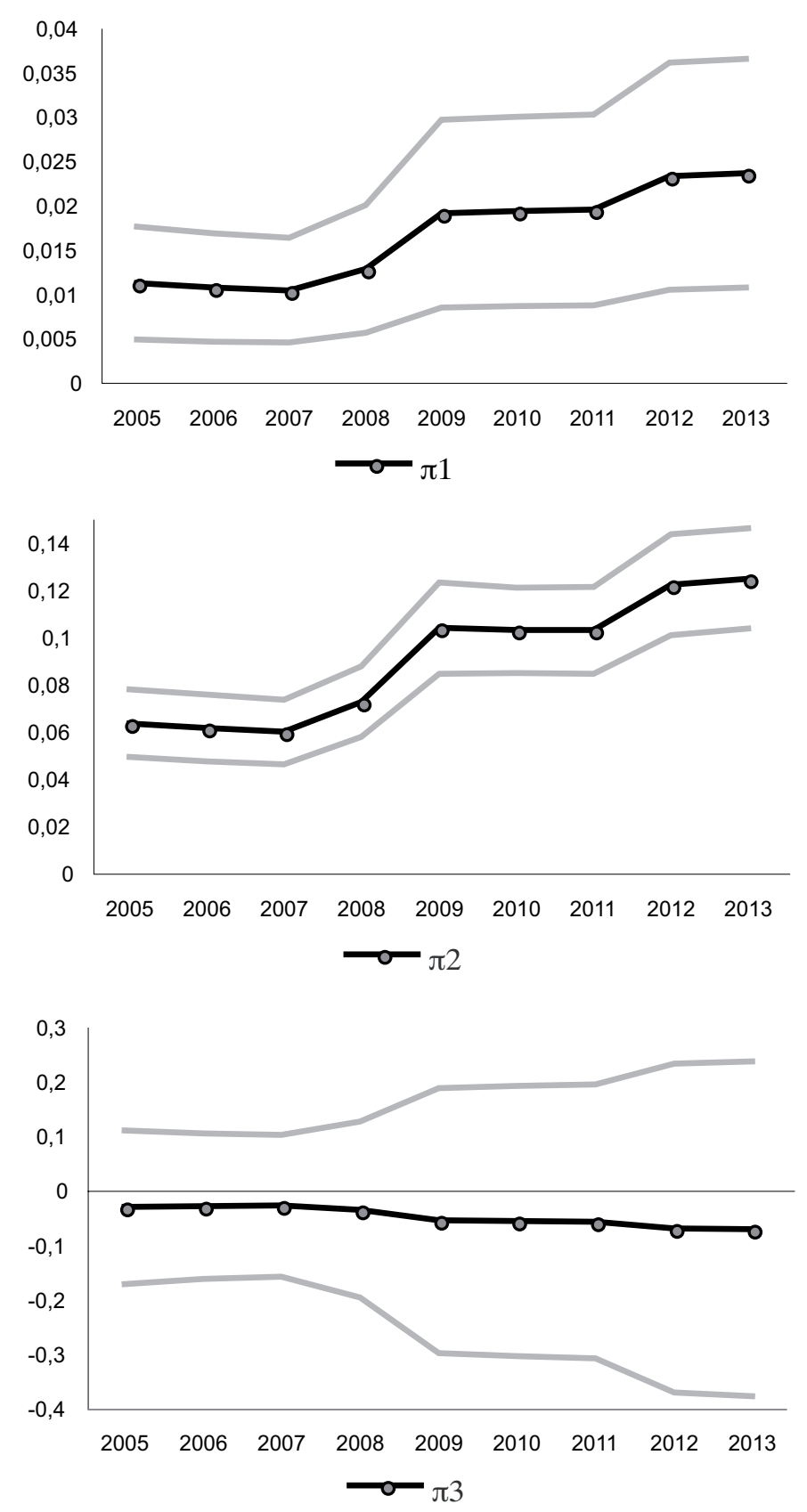
Para analizar con mayor profundidad la evolución temporal del los tres EPMs de interés $\left(\pi_{1}, \pi_{2}, \pi_{3}\right)$, en el Gráfico 3 se muestra la evolución sobre el periodo estudiado de las correspondientes medias anuales obtenidas bajo el modelo probit bivariante dinámico con efectos aleatorios correlacionados (especificación 3). Como se observará, mientras que el EPM de la tasa de vivienda en propiedad regional $\left(\pi_{3}\right)$ permanece no significativamente distinto de cero durante todo el periodo, los EPMs de la forma de tenencia de la vivienda habitual $\left(\pi_{1}\right)$ y de la situación de empleo $\left(\pi_{2}\right)$ anteriores, se incrementan en torno a 1,2 y 6,1 puntos porcentuales, respectivamente, casi doblándose ambos efectos a lo largo de este periodo. Este resultado sugiere que la reciente crisis económica ha intensificado especialmente en España la persistencia de la situación de empleo ${ }^{3}$ y, en menor medida, el efecto positivo que genera sobre el empleo la posesión de la vivienda habitual en propiedad.

Por último, pasamos a estudiar las diferencias que pueden surgir entre propietarios de la vivienda habitual con/sin hipoteca pendiente, en términos de los efectos medios estimados sobre la probabilidad de empleo. Para ello reestimamos los mismos modelos considerados en el Cuadro 3, pero ahora alternativamente excluyendo cada uno de estos tipos de forma de tenencia de la vivienda en propiedad. Nuevamente omitimos el detalle de los resultados por brevedad y pueden ser facilitados bajo petición, recogiéndose en el Cuadro 5 los correspondientes EPMs de interés estimados. Debemos resaltar que ahora las muestras se reducen drásticamente debido a que en la ECV solo se incluyen datos sobre la situación hipotecaria de los propietarios desde el año 2007 en adelante.

En líneas generales, los resultados del Cuadro 5 replican los obtenidos en el Cuadro 3 y, por tanto, se aplican idénticos comentarios salvo dos diferencias principales que se advierten entre los resultados a los que se llega para la muestra I (propietarios sin hipoteca e inquilinos) y II (propietarios con hipoteca e inquilinos). En primer lugar, cuando consideramos la heterogeneidad inobservada en las especificaciones probit 2 y 3 , y confirmado por la especificación lineal GMM 4, la persistencia del empleo $\left(\pi_{2}\right)$ para la muestra II es en torno a 7,5 y 8,5 puntos porcentuales mayor que su contrapartida en la muestra I, apuntando esto a una mejor respuesta ante la crisis económica de los propietarios de vivienda con hipoteca pendiente en términos del mantenimiento de su situación de empleo. Adicionalmente, ser un propietario sin hipoteca en el periodo anterior, en relación con ser inquilino, no altera significativamente la probabilidad de empleo en ninguna de las especificaciones, mientras que el colectivo de propietarios con hipoteca pendiente, en comparación con los inquilinos, manifiesta un incremento significativo de la probabilidad de empleo de casi 2 puntos porcentuales en promedio en las tres primeras especificaciones, desapareciendo este efecto de nuevo en la especificación 4. Para este último modelo cabe recordar las precauciones mencionadas anteriormente, agravadas ahora debido a la notable merma de la muestra utilizada.

${ }^{3}$ Este resultado no es tan sorprendente como pudiera parecer en primera instancia. Lo que está reflejando es que el transcurso de la crisis económica ha provocado que aquellos que permanecen empleados un determinado año tienen cada vez una mayor probabilidad de estar empleados el año siguiente. 


\section{CUADRO 5}

PRINCIPALES EFECTOS PARCIALES MEDIOS SOBRE EL DESEMPLEO DISTINGUIENDO ENTRE PROPIETARIOS CON/SIN HIPOTECA PENDIENTE

\begin{tabular}{|c|c|c|c|c|}
\hline \multicolumn{5}{|c|}{ Muestra I: Propietario sin hipoteca e inquilinos a precio de mercado } \\
\hline & Especificación 1 & Especificación 2 & Especificación 3 & Especificación 4 \\
\hline$\pi_{1}$ & $\begin{array}{c}0,005 \\
(0,011) \\
\end{array}$ & $\begin{array}{c}0,004 \\
(0,012) \\
\end{array}$ & $\begin{array}{c}0,004 \\
(0,009) \\
\end{array}$ & $\begin{array}{c}0,696 \\
(0,338)^{* *}\end{array}$ \\
\hline$\pi_{2}$ & $\begin{array}{c}0,563 \\
(0,018)^{* *}\end{array}$ & $\begin{array}{c}0,079 \\
(0,029)^{* *}\end{array}$ & $\begin{array}{c}0,097 \\
(0,016)^{* *}\end{array}$ & $\begin{array}{c}0,371 \\
(0,055)^{* *}\end{array}$ \\
\hline$\pi_{3}$ & $\begin{array}{l}-0,096 \\
(0,072)\end{array}$ & $\begin{array}{l}-0,105 \\
(0,288) \\
\end{array}$ & $\begin{array}{l}-0,120 \\
(0,255) \\
\end{array}$ & $\begin{array}{l}-1,308 \\
(1,768)\end{array}$ \\
\hline N. ${ }^{o}$ individuos & 4.512 & 4.512 & 4.512 & 2.168 \\
\hline N.o observaciones & 7.745 & 7.745 & 7.745 & 7.596 \\
\hline Log-verosimilitud & $-2.270,75$ & $-2.217,69$ & $-2395,75$ & - \\
\hline \multicolumn{5}{|c|}{ Muestra II: Propietarios con hipoteca pendiente e inquilinos a precio de mercado } \\
\hline & Especificación 1 & Especificación 2 & Especificación 3 & Especificación 4 \\
\hline$\pi_{1}$ & $\begin{array}{c}0,021 \\
(0,009)^{*}\end{array}$ & $\begin{array}{c}0,019 \\
(0,010)^{*}\end{array}$ & $\begin{array}{c}0,017 \\
(0,008)^{*}\end{array}$ & $\begin{array}{c}0,180 \\
(0,176)\end{array}$ \\
\hline$\pi_{2}$ & $\begin{array}{c}0,505 \\
(0,018)^{* *}\end{array}$ & $\begin{array}{c}0,164 \\
(0,035)^{* *}\end{array}$ & $\begin{array}{c}0,172 \\
(0,018)^{* *}\end{array}$ & $\begin{array}{c}0,430 \\
(0,048) * * \\
\end{array}$ \\
\hline$\pi_{3}$ & $\begin{array}{c}-0,143 \\
(0,064)^{*}\end{array}$ & $\begin{array}{l}-0,109 \\
(0,240) \\
\end{array}$ & $\begin{array}{l}-0,112 \\
(0,223) \\
\end{array}$ & $\begin{array}{l}-0,599 \\
(1,139 \\
\end{array}$ \\
\hline N. ${ }^{\circ}$ individuos & 5.113 & 5.113 & 5.113 & 2.538 \\
\hline N. observaciones & 8.930 & 8.930 & 8.930 & 8.893 \\
\hline Log-verosimilitud & $-2.556,71$ & $-2.573,48$ & $-2.899,07$ & - \\
\hline
\end{tabular}

NOTA: Errores estándar entre paréntesis. * Significativo al 5 por 100. ** Significativo al 1 por $100 . \pi_{1}, \pi_{2}$ y $\pi_{3}$ son los EPMs sobre la probabilidad de empleo de, respectivamente, la forma de tenencia de vivienda retardada, la situación de empleo retardado, y la tasa de vivienda en propiedad en la CCAA de residencia.

\section{Conclusiones}

En este trabajo se ha investigado desde un punto de vista empírico el efecto que puede generar la forma de tenencia de la vivienda habitual sobre la situación de empleo de los individuos. Para ello, nos hemos valido del experimento de primera magnitud que ha supuesto la crisis económica en España, y estimamos diferentes modelos dinámicos de panel empleando datos a nivel español a lo largo del periodo 20042013. De los resultados obtenidos podemos destacar tres conclusiones principales.

Primero, si incorporamos explícitamente el efecto de la heterogeneidad inobservada, la endogeneidad de la forma de tenencia de la vivienda como variable explicativa del empleo, y distinguiendo entre la dependencia del estado espuria de la verdadera, la evidencia empírica en España es robusta e indica que no existe ningún efecto 
indirecto de la tasa de propietarios de vivienda regional sobre la probabilidad de empleo de los individuos. Este resultado cuestiona los obtenidos por Blanchflower y Oswald (2013) o Laamanen (2013). En ningún caso en estos últimos trabajos se abordan simultánemente los tres problemas empíricos mencionados.

En segundo lugar, la reciente crisis económica ha intensificado en España la persistencia de la situación de empleo, en mayor medida para los propietarios de vivienda con una hipoteca pendiente, evidenciando este grupo un mayor esfuerzo a la hora de mantener el empleo durante el periodo de crisis.

Por último, el efecto medio de la forma de tenencia de la vivienda habitual sobre la probabilidad de empleo de los individuos es robusto y significativamente positivo, manteniéndose en torno a los 2 puntos porcentuales para el colectivo de propietarios en su conjunto y para los propietarios con hipoteca pendiente, diluyéndose este efecto para los propietarios sin hipoteca. Este resultado está en línea con la evidencia mayoritaria a nivel micro que es concluyente en contra de la hipótesis de Oswald.

En consecuencia, podemos concluir, en contra de la opinión generalizada, que cualquier política de vivienda que persiga la promoción de la vivienda en propiedad es compatible con el objetivo prioritario de reducción del desempleo. O dicho de otra forma, no por mucho fomentar el alquiler, habrá más gente trabajando... 


\section{APÉNDICE}

\section{DESCRIPCIÓN DE LAS VARIABLES EXPLICATIVAS}

\section{CUADRO A1}

DESCRIPCIÓN DE LAS VARIABLES EXPLICATIVAS

\begin{tabular}{|c|c|}
\hline Variables & Definición \\
\hline \multicolumn{2}{|r|}{ Características del hogar } \\
\hline$M I E M B, M I E M B 2$ & Número de miembros del hogar y su cuadrado \\
\hline \multicolumn{2}{|r|}{ Características del responsable de la vivienda } \\
\hline SEXO & Femenino $=0 ;$ masculino $=1$. \\
\hline PAREJA & $\begin{array}{l}\text { Casado o viviendo en pareja en el mismo hogar }(\text { con o sin base legal })=1 \text {; } \\
\text { resto }=0 \text {. }\end{array}$ \\
\hline$S A L U D$ & $\begin{array}{l}\text { Estado de salud reportado por el individuo excelente, bueno o normal=1, } \\
\text { resto }=0 \text {. }\end{array}$ \\
\hline NACESP & Nacido en España $=1$, resto $=0$ \\
\hline Edad: & \\
\hline$E D A D$ & Edad del individuo. \\
\hline$E D A D 25 *$ & Edad del individuo menor de 25 años $=1$; resto $=0$. \\
\hline$E D A D 35$ & Individuo entre 25 y 34 años $=1$; resto $=0$. \\
\hline$E D A D 45$ & Individuo entre 35 y 44 años $=1$; resto $=0$. \\
\hline EDAD55 & Individuo entre 45 y 54 años $=1$; resto $=0$. \\
\hline$E D A D 55+$ & Individuo por encima de 54 años $=1$; resto $=0$. \\
\hline Educación: & \\
\hline$E D U 1 *$ & Individuo sin estudios, con estudios primarios o que ha abandonado la \\
\hline EDU2 & educación secundaria sin completarla=1; resto $=0$. \\
\hline EDU3 & $\begin{array}{l}\text { Individuo con estudios de educación secundaria o de formación e inserción } \\
\text { laboral que precise título de segunda etapa de secundaria }=1 \text {; resto }=0 \text {. } \\
\text { Individuo con estudios de educación superior }=1 \text {, resto }=0 \text {. }\end{array}$ \\
\hline $\begin{array}{l}\text { Datos laborales: } \\
N T R A B, N T R A B 2\end{array}$ & $\begin{array}{l}\text { Número total de años pasados en trabajo remunerado en toda la vida laboral } \\
\text { del individuo, y su cuadrado. }\end{array}$ \\
\hline \multicolumn{2}{|c|}{ Características del área local o de la comunidad autónoma de residencia } \\
\hline$D E N S P O B$ & $\begin{array}{l}\text { El área de residencia es un conjunto contiguo de áreas locales donde cada uno } \\
\text { tiene una densidad superior a } 500 \text { habitantes por } \mathrm{km}^{2} \text {, y donde la población } \\
\text { total es al menos de } 50000 \text { habitantes }=1 \text {, resto }=0\end{array}$ \\
\hline TUNIFAM & $\begin{array}{l}\text { Porcentaje de hogares viviendo en viviendas unifamiliares aisladas o ado- } \\
\text { sadas en la comunidad autónoma de residencia obtenido a partir de la ECV. } \\
\text { (Fuente: I.N.E. }\end{array}$ \\
\hline$T P D A D$ & $\begin{array}{l}\text { Porcentaje de vivienda en propiedad en la comunidad autónoma de residencia } \\
\text { obtenida a partir de la ECV. (Fuente: INE) }\end{array}$ \\
\hline TPARO & $\begin{array}{l}\text { Tasa de desempleo }(\%) \text { en la comunidad autónoma de residencia obtenida a } \\
\text { partir de la Encuesta de Población Activa (EPA). Fuente: INE. }\end{array}$ \\
\hline \multicolumn{2}{|r|}{ Indicadores de periodo } \\
\hline $\begin{array}{l}A 04 *, A 05, A 06, A 07 \\
\mathrm{~A} 08, \mathrm{~A} 09, \mathrm{~A} 10, \mathrm{~A} 11 \\
A 12, A 13\end{array}$ & Variables binarias indicadoras del año de observación. \\
\hline
\end{tabular}

NOTA: * Variable de referencia. 
CUADRO A2

ESTADÍSTICOS DESCRIPTIVOS PARA LA MUESTRA TOTAL

\begin{tabular}{|c|c|c|c|c|c|c|c|}
\hline & \multicolumn{2}{|c|}{ Total } & \multicolumn{2}{|c|}{ Propietarios } & \multicolumn{2}{|c|}{ Inquilinos } & \multirow{2}{*}{$\begin{array}{l}\text { Diferencia } \\
\text { medias }^{1}\end{array}$} \\
\hline & Medias & $\begin{array}{c}\text { Desv. } \\
\text { estándar }\end{array}$ & Medias & $\begin{array}{c}\text { Desv. } \\
\text { estándar }\end{array}$ & Medias & $\begin{array}{c}\text { Desv. } \\
\text { estándar }\end{array}$ & \\
\hline EMPLEO & 0,888 & 0,316 & 0,895 & 0,306 & 0,821 & 0,383 & $0,074 * *$ \\
\hline MIEMB & 3,181 & 1,273 & 3,205 & 1,227 & 2,969 & 1,607 & 0,236 ** \\
\hline MIEMB2 & 11,737 & 9,063 & 11,776 & 8,503 & 11,395 & 12,968 & $0,381 * *$ \\
\hline SEXO & 0,701 & 0,458 & 0,710 & 0,454 & 0,628 & 0,483 & $0,081 * *$ \\
\hline PAREJA & 0,765 & 0,424 & 0,785 & 0,411 & 0,589 & 0,492 & $0,196 * *$ \\
\hline SALUD & 0,972 & 0,166 & 0,972 & 0,166 & 0,971 & 0,167 & 0,001 \\
\hline NACESP & 0,916 & 0,278 & 0,956 & 0,205 & 0,560 & 0,496 & 0,396 ** \\
\hline$E D A D$ & 45,726 & 9,265 & 46,264 & 9,046 & 41,016 & 9,815 & $5,248 * *$ \\
\hline$E D A D 25$ & 0,005 & 0,068 & 0,002 & 0,048 & 0,024 & 0,153 & $-0,022 * *$ \\
\hline EDAD35 & 0,127 & 0,333 & 0,110 & 0,313 & 0,273 & 0,446 & $-0,162^{* * *}$ \\
\hline EDAD45 & 0,317 & 0,465 & 0,315 & 0,465 & 0,335 & 0,472 & $-0,020$ *** \\
\hline EDAD55 & 0,349 & 0,477 & 0,359 & 0,480 & 0,263 & 0,440 & 0,096 ** \\
\hline EDAD55+ & 0,202 & 0,402 & 0,213 & 0,410 & 0,105 & 0,307 & $0,108 * *$ \\
\hline$E D U 1$ & 0,169 & 0,374 & 0,170 & 0,376 & 0,155 & 0,362 & $0,015^{* *}$ \\
\hline$E D U 2$ & 0,484 & 0,500 & 0,479 & 0,500 & 0,530 & 0,499 & $-0,051 * *$ \\
\hline$E D U 3$ & 0,347 & 0,476 & 0,351 & 0,477 & 0,315 & 0,464 & $0,036^{* * *}$ \\
\hline NTRAB & 23,743 & 10,718 & 24,342 & 10,582 & 18,493 & 10,473 & $5,849 * *$ \\
\hline NTRAB2 & 678,582 & 546,362 & 704,487 & 549,165 & 451,658 & 462,916 & $252,829 * *$ \\
\hline DENSPOB & 0,501 & 0,500 & 0,494 & 0,500 & 0,558 & 0,497 & $-0,063 * *$ \\
\hline TUNIFAM & 0,365 & 0,165 & 0,367 & 0,165 & 0,352 & 0,160 & 0,015 ** \\
\hline$T P D A D$ & 0,795 & 0,053 & 0,797 & 0,051 & 0,779 & 0,061 & 0,017 ** \\
\hline TPARO & 0,155 & 0,076 & 0,155 & 0,077 & 0,155 & 0,074 & 0,000 \\
\hline$A 04$ & 0,052 & 0,221 & 0,052 & 0,223 & 0,045 & 0,207 & $0,007 *$ \\
\hline$A 05$ & 0,090 & 0,286 & 0,091 & 0,288 & 0,080 & 0,271 & $0,012 *$ \\
\hline A06 & 0,092 & 0,289 & 0,093 & 0,290 & 0,086 & 0,281 & 0,007 \\
\hline$A 07$ & 0,109 & 0,312 & 0,110 & 0,313 & 0,106 & 0,308 & 0,003 \\
\hline A08 & 0,123 & 0,329 & 0,124 & 0,329 & 0,119 & 0,324 & 0,004 \\
\hline A09 & 0,128 & 0,334 & 0,128 & 0,334 & 0,133 & 0,339 & $-0,005$ \\
\hline A10 & 0,112 & 0,315 & 0,111 & 0,314 & 0,121 & 0,326 & $-0,011 *$ \\
\hline$A 11$ & 0,100 & 0,300 & 0,099 & 0,299 & 0,108 & 0,310 & $-0,008$ \\
\hline A12 & 0,114 & 0,318 & 0,114 & 0,318 & 0,119 & 0,324 & $-0,006$ \\
\hline A13 & 0,079 & 0,270 & 0,079 & 0,269 & 0,083 & 0,275 & $-0,004$ \\
\hline Individuos & 15.169 & & & & & & \\
\hline Obser. totales & 42.085 & & & & & & \\
\hline
\end{tabular}

NOTAS: ${ }^{1}$ Diferencia entre las medias de propietarios e inquilinos (a precio de mercado) de vivienda. * significativo al 5 por 100. ** significativo al 1 por 100. 


\section{Referencias bibliográficas}

[1] ALESSIE, R.; HOCHGUERTEL, S. y VAN SOEST, A. (2004). «Ownership of stocks and mutual funds: A panel data analysis». The Review of Economics and Statistics, 86 (3), 783-796.

[2] ARELLANO, M. y BOND, S. (1991). «Some tests of specification for panel data: Monte Carlo evidence and an application to employment equations». Review of Economic Studies, 58, 277-297.

[3] BARRIOS, J. A. y RODRÍGUEZ, J. E. (2004). «User cost changes, unemployment and home-ownership: Evidence from Spain». Urban Studies, 41 (3), 563-578.

[4] BATTU, H.; MA, A. y PHIMISTER, E. (2008). «Housing tenure, job mobility and unemployment in the UK». The Economic Journal, 118, 311-328.

[5] BLANCHFLOWER, D. G. y OSWALD, A. J. (2013). «Does high home-ownership impair the labor market?». NBER Working Paper No. 19079, Cambridge, MA.

[6] BLUNDELL, R. y BOND, S. (1998). «Initial conditions and moment restrictions in dynamic panel data models». Journal of Econometrics, 87, 115-143.

[7] BRUNET, C. y LESUEUR, J. (2009). «Do homeowners stay unemployed longer? A French microeconometric study». En C. van Ewijk y M. van Leuvenjstein (eds.), Homeownership and the Labour Market in Europe. New York: Oxford University Press, 137-160.

[8] CHAMBERLAIN, G. (1980). «Analysis of covariance with qualitative data». The Review of Economic Studies, 47, pp. 225-238.

[9] COULSON, N. E. y Fisher, L. M. (2002). «Tenure choice and labour market outcomes». Housing Studies, 17, 35-49.

[10] COULSON, N. E. y FISHER, L. M. (2009). «Housing tenure and labor market impacts: The search goes on». Journal of Urban Economics, 65 (3), 252-264.

[11] COULSON, N. E. y GRIECO, P. L. (2013). «Mobility and mortgages: Evidence from the PSID». Regional Science and Urban Economics, 43, 1-7.

[12] DELLA VIGNA, S. y PASERMAN, M. D. (2005). «Job search and impatience» . Journal of Labor Economics, 23 (3), 527-588.

[13] DOHMEN, T. J. (2005). «Housing mobility and unemployment». Regional Science and Urban Economics, 35, 305-325.

[14] DUJARDIN, C. y GOFFETTE-NAGOT, F. (2010). «Neighborhood effects on unemployment? A test à la Altonji». Regional Science and Urban Economics, 40, 380-396.

[15] FARBER, H. S. (2012). «Unemployment in the great recession: Did the housing market crisis prevent the unemployed from moving to take jobs?».American Economic Review, 102 (3), 520-525.

[16] FlATAU, P.; FORBES, M.; HENDERSHOTT, P. H. y WOOD, G. (2003). «Homeownership and unemployment: The roles of leverage and public housing», NBER Working Paper No. 10021, Cambridge, MA.

[17] GREEN, R. K. y HENDERSHOTT, P. H. (2001). «Home-ownership and unemployment in the US». Urban Studies, 38 (9), 1509-1520.

[18] HEAD, A. y LLOYD-ELLIS, H. (2012). «Housing liquidity, mobility and the labour market». Review of Economic Studies, 79 (4), 1559-1589.

[19] ISEBAERT, D.; HEYLEN, F. y SMOLDER, C. (2015). «Houses and/or jobs: ownership and the labour market in Belgian districts». Regional Studies, 49 (8), 1387-1406. 
[20] IOANNIDES, Y. M. y LOURY, L. D. (2004). «Job information networks, neighborhood effects, and inequality». Journal of Economic Literature, XLII, 1056-1093.

[21] JAMES, R. N. (2009). «Tenant time preference as a barrier to homeownership». Applied Economics Letters, 16, 1073-1077.

[22] LAAMANEN, J.-P. (2013). «Home ownership and the labour market: Evidence from rental housing market deregulation». Tampere Economic Working Paper, No. 89, University of Tampere, Finland. Descargado en septiembre 2015 de http://tampub.uta. fi/handle/10024/68116.

[23] MORTENSEN, D. T. (1986). «Job search and labour market analysis». En O. Ashenfelter y D. Card (eds.), Handbook of Labor Economics, II, Amsterdam: North Holland, 849-919.

[24] MUNCH, J. R.; ROSHOLM, M. y SVARER, M. (2006). «Are homeowners really more unemployed?». The Economic Journal, 116, 991-1013.

[25] MUNCH, J. R.; ROSHOLM, M. y SVARER, M. (2008). «Home ownership, job duration and wages». Journal of Urban Economics, 63, 130-145.

[26] MUNDLAK, Y. (1978). «On the pooling of time series and cross section data». Econometrica, 46, 69-85.

[27] NICKELL, S. (1998). «Unemployment: questions and some answers». The Economic Journal, 108, 802-816.

[28] NICKELL, S. y LAYARD, R. (1999). «Labor market institutions and economic performance». En O. Ashenfeiter y D. Card (eds.), Handbook of Labor Economics, 3c, New York: North Holland, 3029-3084.

[29] OSWALD, A. J. (1996). «A conjecture on the explanation for high unemployment in the industrialized nations: Part I», Warwick Economics Research Paper, \#475, University of Warwick.

[30] OSWALD, A. J. (1999). The housing market and Europe's unemployment: A nontechnical paper, mimeo, University of Warwick.

[31] PICCHIO, M. y VAN OURS, J. C. (2013). «Retaining through training even for older workers». Economics of Education Review, 32, 29-48.

[32] PISSARIDES, C. A. (2000). Equilibrium Unemployment Theory, Cambridge: MIT Press.

[33] RODRÍGUEZ, J. E. y BARRIOS, J. A. (2012). «¿Incide la forma de tenencia de la vivienda habitual sobre la situación de empleo en España?». Estudios de Economía Aplicada, 30 (2), 751 (22 págs.), versión electrónica en www.revista-eea.net, ref. ə30201 .

[34] RODRÍGUEZ, J. E. y BARRIOS, J. A. (2013). «Una estimación del efecto de la forma de tenencia de la vivienda sobre el empleo en España». Estadística Española, 55 (181), 187-212.

[35] ROUWENDAL, J. y NIJKAMP, P. (2010). «Homeownership and labour market behaviour: Interpreting the evidence”. Environment and Planning A, 42 (2), 419-433.

[36] STEWART, M. B. (2007). «The interrelated dynamics of unemployment and low-wage employment». Journal of Applied Econometrics, 22, 511-531.

[37] VAN EWIJK, C. y VAN LEUVENJSTEIN, M. (2009). Homeownership and the Labour Market in Europe. New York: Oxford University Press.

[38] WOOLDRIDGE, J. M. (2005). «Simple solutions to the initial conditions problem for dynamic, nonlinear panel data models with unobserved heterogeneity». Journal of Applied Econometrics, 20, 39-54. 